\title{
Mechanisms of sex differentiation and sex reversal in hermaphrodite fish revealed by Epinephelus coioides genome
}

\author{
Yong Zhang ${ }^{1}$, Shuisheng Li $^{1}$, Wanshun $\mathrm{Li}^{1}$, Shoujia Jiang ${ }^{1}$, Yi Jing ${ }^{1}$, Ling Xiao ${ }^{1}$, Yangyang \\ $\mathrm{Yu}^{1}$, Yun Liu ${ }^{1}$, Yanhong $\mathrm{Li}^{1}$, Dengdong Wang ${ }^{1}$, Jiang Li ${ }^{1}$, Cheng Peng ${ }^{1}$, Jiaxing Chen ${ }^{1}$, \\ Dan $\mathrm{Lu}^{1}$, Bin $\mathrm{Wu}^{2}$, X Guang ${ }^{3}$, Junping $\mathrm{Ma}^{1}$, Xinxin You ${ }^{1}$, Yuqing Yang ${ }^{1}$, Su Liu ${ }^{1}$, \\ Xiaodong Fang ${ }^{3}$, Q Gao ${ }^{3}$, Qiong Shi ${ }^{1}$, Haoran Lin $^{1}$, Manfred Schartl ${ }^{4}$, and Yue Zhen ${ }^{1}$ \\ ${ }^{1}$ Affiliation not available \\ ${ }^{2} \mathrm{BGI}$ \\ ${ }^{3}$ BGI-Shenzen \\ ${ }^{4}$ University of Wurzburg
}

March 8, 2022

\begin{abstract}
Abstract: Most grouper species are functional protogynous hermaphrodites, but the genetic basis and the molecular mechanisms underlying regulation of this unique reproductive strategy remain enigmatic. In this study, we reported a high-quality chromosome-level genome assembly of the representative orange-spotted grouper (Epinephelus coioides). No duplication or deletion of sex differentiation related genes was found in the genome, suggesting that sex development in this grouper may be related to changes in regulatory sequences or environmental factors. Transcriptomic analyses showed that aromatase and retinoic acid are probably critical to promote ovarian fate determination, and follicle-stimulating hormone triggers the femaleto-male sex change. Socially controlled sex-change studies revealed that the brain of sex-changing fish in response to social environment may be mediated by activation of phototransduction cascade and the melatonin synthesis pathway. In summary, our genomic and experimental results provide novel insights into the molecular mechanisms of sex differentiation and sex change in the protogynous grouper.
\end{abstract}

\section{Hosted file}

Grouper manuscript.doc available at https://authorea.com/users/464093/articles/559061mechanisms-of-sex-differentiation-and-sex-reversal-in-hermaphrodite-fish-revealed-byepinephelus-coioides-genome 

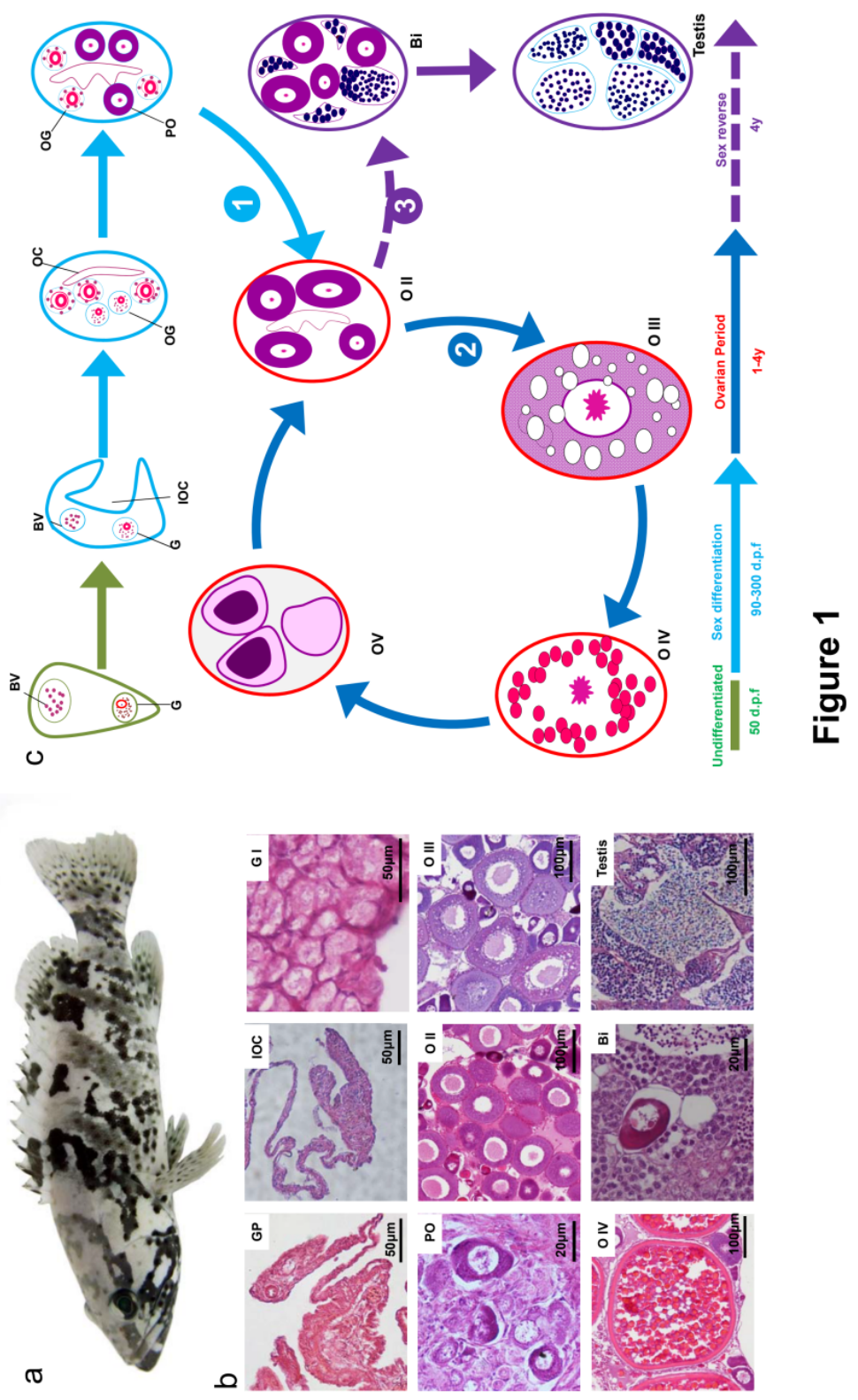

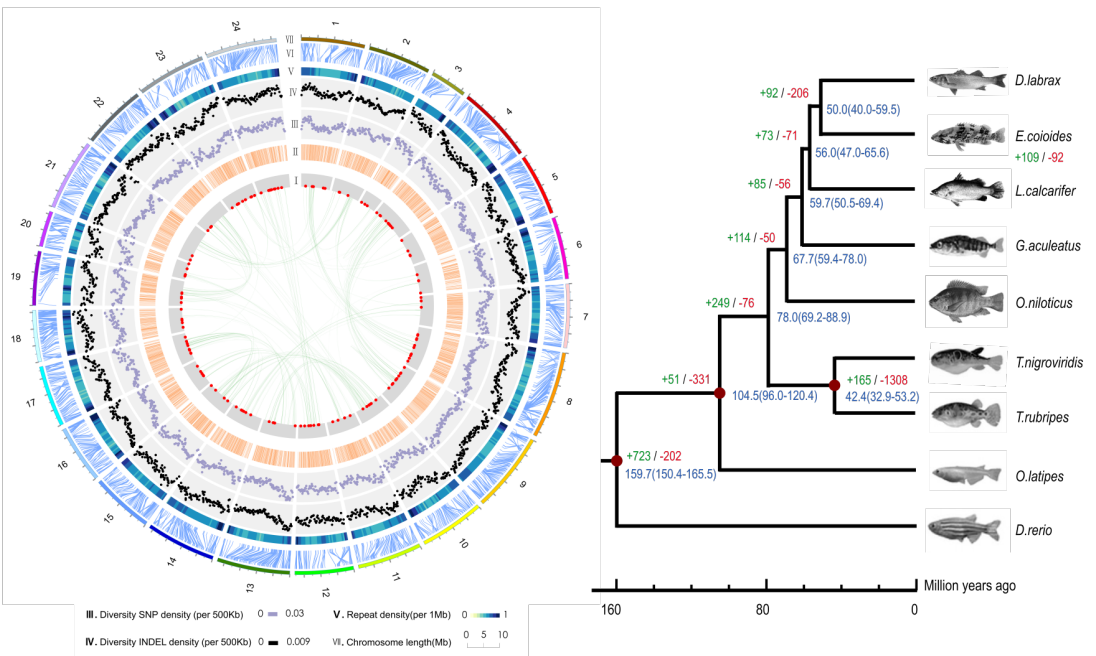

Figure 2 

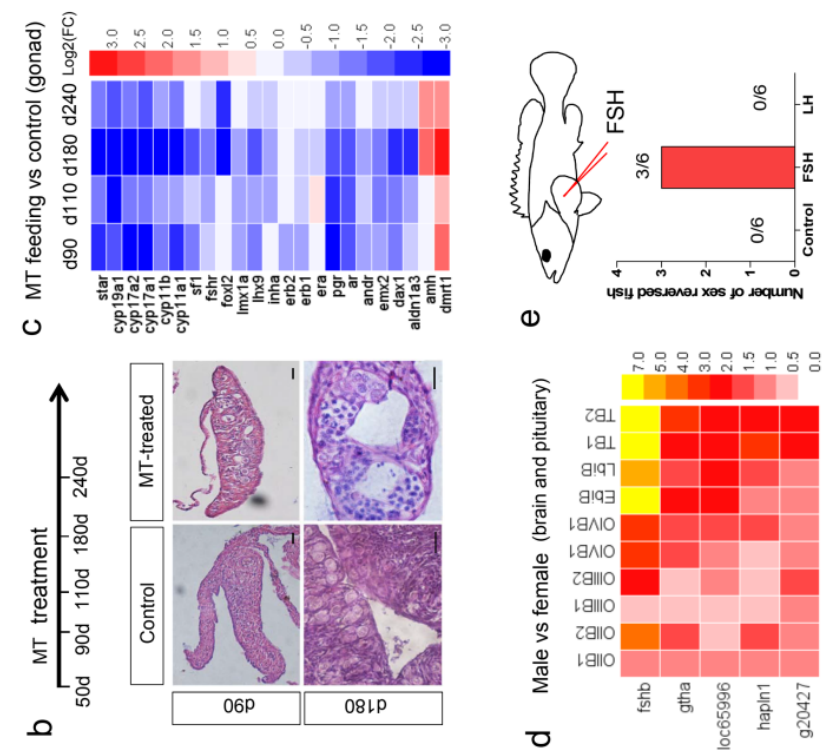

인

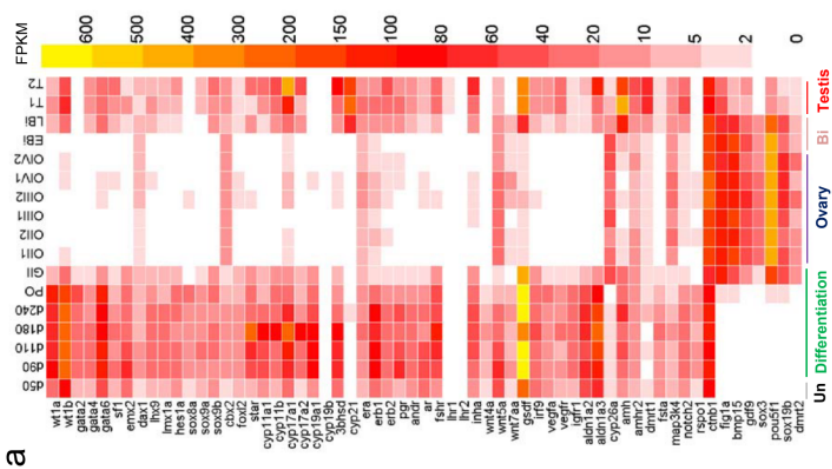


a

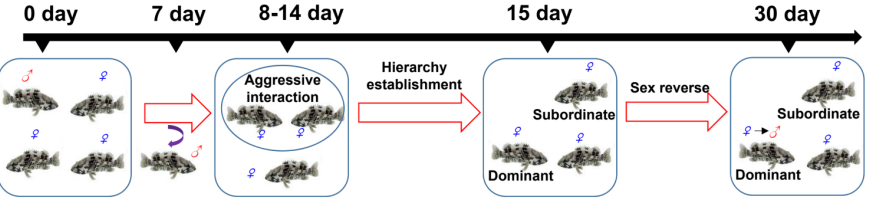

b

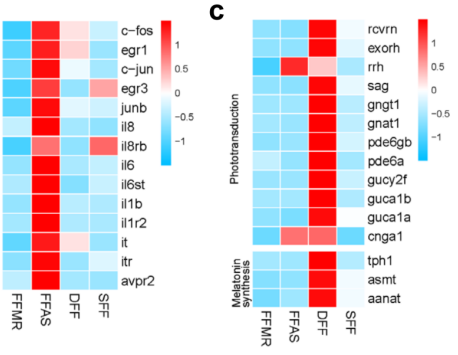

d

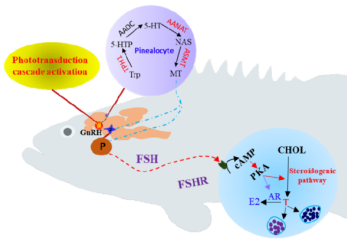

Figure 4

\section{Hosted file}

Supplementary Text and Figures.docx available at https://authorea.com/users/464093/ articles/559061-mechanisms-of-sex-differentiation-and-sex-reversal-in-hermaphroditefish-revealed-by-epinephelus-coioides-genome 